\title{
Estrategias iniciales en el manejo de pacientes con dispepsia
}

\begin{abstract}
Escenario clínico:
Un varón de 32 años consulta a su médico de familia por síntomas de dispepsia de varios meses de evolución. No tiene antecedentes de importancia ni síntomas de alarma para sospechar cáncer.

El médico sabe que existen varias alternativas propuestas para el manejo de estos pacientes. Una de las posibilidades es realizar endoscopía (FEDA), otra es indicar tratamiento previa realización de test para detectar helicobacter pylori (HP) y proceder de acuerdo con el resultado, y otra es realizar directamente tratamiento empírico. En este paciente nos interesó principalmente compara FEDA inicial o detección de HP y luego tratamiento en base al resultado.
\end{abstract}

\section{Pregunta que generó el caso:}

En los pacientes con dispepsia (población) la realización de FEDA inicial (intervención) comparada con la estrategia de determinar presencia de HP (comparación) vs tratamiento empírico inicial es superior para disminuir el período sintomático (resultado)?

\section{Estrategia de búsqueda:}

Se realizó una búsqueda en MEDLINE empleando los términos non ulcer dyspepsia, management strategies. Se restringió la búsqueda a ensayos aleatorizados en adultos y publicados en los últimos 15 años. Se obtuvieron 15 citas. Se seleccionaron dos que parecían apropiadas para responder la pegunta.

\section{Dispepsia:}

La dispepsia constituye un costoso y frecuente problema en Atención Primaria. Las causas principales son: dispepsia funcional $(50 \%)$, enfermedad ulcerosa (20\%), reflujo gastroesofágico (20\%) y cáncer gástrico $(<2 \%)$. Sin embargo, la estrategia inicial más efectiva o costo-efectiva es motivo de debate y controversia.

Algunos profesionales prefieren el uso temprano de la FEDA en todos los pacientes mientras que otros prefieren reservar la FEDA para pacientes con síntomas de alarma o que fracasan con el tratamiento empírico.

Estudios previos1 han mostrado que la FEDA temprana es una aproximación más costo-efectiva que el tratamiento con antagonistas $\mathrm{H} 2$ y genera mayor satisfacción en los pacientes. Sin embargo, con el reconocimiento del papel que juega el Helicobacter ha comenzado a alterar estas conductas.2-3 En los países desarrollados entre el 25 y $50 \%$ de la población con dispepsia tiene helicobacter ${ }^{4}$ de los cuales entre un tercio y la mitad tiene enfermedad ulcerosa.

Modelos de decisión han sugerido que la estrategia de determinar la infección por helicobacter de manera no invasiva y su erradicación es más costo-efectiva que la FEDA temprana. ${ }^{5}$

La terapia de erradicación del helicobacter curará las úlceras y resolverá sus síntomas y podría mejorar los síntomas en un pequeño grupo de pacientes con dispepsia. 6

\section{Resumen de la evidencia:}

Lassen At, Pedersen FM, Bytzer P, Schaffalitzky de Muckadell OB. Helicobacter test-and-eradicate versus prompt endoscopy for management of dyspeptic patients: a randomised trial. Lancet 2000; 356: 455-60.

576 pacientes con dispepsia con una edad promedio de 45 años fueron reclutados a través de médicos de atención primaria de Dinamarca. Luego de excluir a los que presentaban comorbilidad importante como hemorragia digestiva alta, 500 pacientes fueron aleatorizados y 447 completaron un año de seguimiento.

\section{Diseño del estudio:}

Este fue un ensayo aleatorizado y controlado que evaluó dos estrategias para el manejo de pacientes con dispepsia: endoscopía precoz vs realización de test para evaluar presencia de HP y proceder de acuerdo con el resultado. La aleatorización se hizo con una tabla de números aleatorizados. Los grupos fueron similares al inicio del ensayo, a pesar de que el grupo "endoscopía precoz" presentaba una historia más prolongada de dispepsia. Los pacientes eran entrevistados al mes y al año. Además completaban un diario de síntomas una semana por mes durante el año de seguimiento.

Pacientes aleatorizados al grupo test para HP: a todos los pacientes de esta rama se les realizaba un test de urea para determinar el status de la infección por HP. Los que tenían un test positivo recibieron tratamiento con lansoprazol $30 \mathrm{mg}$, amoxicilina y metronidazol por dos semanas. Los que tenían HP negativo y síntomas de reflujo recibieron inhibidores de la bomba de protones por un mes y luego "a demanda". Aquellos pacientes que no mejoraban o recaían, se les realizaba endoscopía (FEDA). Los pacientes con HP negativo, pero con historia de consumo de antiinflamtorios recibían FEDA.

Pacientes aleatorizados a FEDA precoz: este grupo fue tratado de acuerdo con los hallazgos endoscópicos. Los que presentaron úlcera duodenal recibieron tratamiento para HP seguido de dos semanas de inhibidores de la bomba de protones (IBP). Los pacientes con úlceras gástricas fueron tratados acorde al status con relación al HP. Las úlceras gástricas fueron biopsiadas cada seis semanas hasta la cicatrización. Los pacientes con esofagitis por reflujo (ER) fueron tratados con IBP durante 8 semanas. Los pacientes con dispepsia funcional recibieron medidas no farmacológicas y reaseguro. Se les indicaron IBP si el paciente los había consumido con éxito en el pasado.

Medición de resultados: el resultado principal fue porcentaje de días sin síntomas de dispepsia. Resultados secundarios: calidad de vida (medida con escala análoga y una escala de rating de síntomas gastrointestinales), visitas al médico, internaciones, satisfacción, uso de FEDA y empleo de IBP.

Resultados: no se encontraron diferencias en la proporción de días sin dispepsia, puntajes de calidad de vida, visitas al médico ni en el número de internaciones. Hubo una diferencia significativa en el porcentaje de pacientes que reportaron mejoría de los sintomas al mes a favor del grupo FEDA precoz (28\% vs $20 \%)$. Al año, no hubo diferencias.

Los pacientes asignados a la estrategia test HP-tratamiento estaban más insatisfechos al año ( $12 \%$ vs $4 \%)(p=0.013)$. Como era de esperar, el empleo de FEDA fue mayor en el grupo FEDA precoz ( 0.5 vs 1.25 por persona, $p<0.0001$ ).

Conclusión: la estrategia de test HP-erradicación es eficiente y segura para el manejo de pacientes con dispepsia en atención primaria, a pesar de que los pacientes están más satisfechos con la realización de FEDA precoz.

Delaney BC, Wilson S, Roalfe A, Redman V, Wearn A, Hobbs FDR. 
Randomised controlled trial of Helicobacter pylori testing and endoscopy for dyspepsia in primary care. BMJ 2001, 332: 1- 5

Objetivo del estudio: determinar la efectividad y costo efectividad de la estrategia rastreo de HP y endoscopía vs tratamiento empírico inicial en pacientes con dispepsia.

\section{Diseño: ensayo aleatorizado y controlado:}

Ambito: 31 centros de Atención Primaria del Reino Unido.

Pacientes: se reclutaron 478 pacientes menores de 50 años que consultaron por dispepsia de más de 4 semanas de duración a médico general.

Intervención: los pacientes fueron asignados de manera aleatorizada a una estrategia que consistía en determinar la infección por HP y acceso abierto a la FEDA vs tratamiento habitual. Los pacientes en el grupo intervención con HP positivos eran derivados para realización de FEDA. Los que presentaban HP negativo eran tratados con supresores de la secreción ácida a elección de médico general. Los pacientes del grupo control fueron manejados de manera habitual. El manejo habitual contemplaba la posibilidad de derivar al gastroenterólogo, pero el médico no podía acceder abiertamente a la FEDA como manejo inicial. Los pacientes cuyo tratamiento fracasaba, podían realizar FEDA a las 6 semanas.

Medición de resultados: el resultado principal fue la efectividad medida por los síntomas y los costos del manejo de la patología. Los síntomas se midieron al inicio del ensayo y al año y medio con la escala de Birmingham para dispepsia.

Para los costos se tomaron en cuenta los recursos empleados en atención primaria y secundaria los 12 meses siguientes a la aleatorización.

Resultados: los datos fueron analizados por intención de tratar y al final del ensayo se contaba con información del $99 \%$ de los pacientes aleatorizados. Un $40 \%$ de los pacientes en la rama intervención tuvieron test para HP positivo. El $45 \%$ de los pacientes en el grupo intervención recibieron FEDA vs el $25 \%$ en el grupo control. Ningún paciente del grupo control recibió la estrategia del grupo interevnción.

De los pacientes que realizaron FEDA, se detectaron significativamente más úlceras en el grupo test HP-FEDA que en el control ( $7 \%$ vs $2.1 \%, p=0.0011)$

No hubo diferencias entre los grupos en los puntajes de mejoría sintomática y calidad de vida. No hubo diferencias en el consumo de medicación para la dispepsia. Tampoco hubo diferencias en el número de consultas ni en la indicación de regímenes para erradicación del HP. La estrategia test HP-FEDA tuvo un costo de 367.85 libras por paciente esterlinas vs 253.16 del grupo control.

Conclusión: la aplicación de una estrategia de testear para HP -FEDA aumenta la indicación de FEDA del 25\% (cuidado usual) al $40 \%$. Sin embargo esta estrategia no produce diferencias significativas en los síntomas ni en la calidad de vida comparada con el cuidado estándar. El aumento en los costos sin mejoría en los resultados no justifica su aplicación.

\section{Comentario y recomendación para la práctica:}

Las fortalezas de estos estudio incluyen: el diseño aleatorizado, alta tasa de seguimiento al año y el hecho de que los pacientes hayan sido reclutados en Centros de Atención Primaria. Además estos estudios intentan reproducir la conducta habitual con estos pacientes acercándose bastante a lo que sucede en la vida real. Los resultados medidos están focalizados en los pacientes y no en la enfermedad como podría haber sido la medición de la erradicación del HP o la tasa de curación de úlceras.

En el primer estudio, no queda claro si los evaluadores estaban "ciegos" a la estrategia que recibía el paciente.

Los resultados pueden aplicarse a la Atención Primaria dado que los pacientes fueron reclutados en este nivel de atención.

Al seir estudios "negativos", es decir que no haya diferencias en el resultado principal evaluado, uno debe asegurarse que el tamaño de la muestra haya sido suficiente para encontrar diferencias, de haber existido. Ambos estudios tuvieron un poder ${ }^{\star}$ del $90 \%$ para detectar las diferencias clínicamente relevantes entre ambos grupos, por lo que este aspecto no debe intranquilizarnos.

Si bien en el estudio del Lancet la edad promedio fue de 45 años, el rango abarcó desde 18 a 88 años. La cantidad de pacientes no permitió realizar análisis en el subgrupo de pacientes mayores de 65 años.

Sin embargo, deberíamos ser cautelosos a la hora de instaurar una estrategia de test para HP-tratamiento en adultos mayores debido a la diferente prevalencia de infección por HP, cáncer y consumo de antiinflamatorios. De acuerdo con la información publicada, ${ }^{7}$ de aplicarse esta estrategia en ancianos, $50 \%$ de los pacientes se habrían tratado para erradicar el HP a pesar de no tener indicación; y pacientes sin HP, ni reflujo ni historia de consumo de AINEs habrían recibido tratamiento no farmacológico y reaseguro a pesar de presentar patología. Debemos aclarar que los estudios en ancianos no han sido realizados en el ámbito de la Atención Primaria. Hasta que este punto se aclare, podemos concluir que la estrategia de test HP-tratamiento es eficaz y segura para pacientes con dispepsia atendidos por médicos generales y puede ser empleada. Debido a que la satisfacción con esta estrategia es menor, la educación y esclarecimiento por parte del médico son fundamentales para no deteriorar el vínculo y la relación médico-paciente. Esta estretegia (test HP-tratamiento) es muy útil para lugares donde el acceso a la FEDA está restringido por costos, disponibilidad o aceptabilidad. Lamentablemente, no contamos hasta la fecha con un mismo ensayo que haya evaluado las tres estrategias simultáneamente. La estrategia de tratar de manera empírica a los menores de 50 años es efectiva para el alivio de los síntomas y más económica que testear para HP-FEDA. La preocupación de implementar esta estrategia se relaciona con el hecho de "perdernos" la posibilidad de erradicar al HP en pacientes que presentan úlcera recurrente por la infección. La erradicación del HP podría ser útil en algún subgrupo de pacientes con dispepsia no ulcerosa. Queda por aclarar en qué subgrupo de pacientes con dispepsia la estrategia test HP-FEDA es costo efectiva en Atención Primaria comparada con tratamiento empírico.

A pesar que no hay estudios que comparen las tres estrategias a la vez, las tres parecerían ser similarmente efectivas en menores de 50 para el manejo de los síntomas. Su diferenciación radica más en la posible satisfacción del paciente y en el costo que implican al sistema de salud.

Dra. Karin Kopitowski [ Directora de Atención Médica. Unidad de Medicina Familiar y Preventiva. Hospital Italiano de Buenos Aires ]

\section{Referencias}

1. Bytzer P, Hansen JM, Schaffalitzky, et al. Empirical H2 blocker therapy or prompt endoscopy in management of dyspepsia. Lancet 1994;343:811-16

2. Sobala GM, Crabtree JE, Penit JA, et al. Screening dyspepsia by serology to Helicobacter pylori. Lancet 1991; 338:94-96.

3. Anon. American Gastroenterological Association medical position statement: evaluation of dyspepsia. Gastroenterology 1998; 114:579-81.

4. Agreus L, Engstrand L, Svardsudd K, et al. Helicobacter pylori seroposivity among swedish adults with and without abdominal symptoms: a population based epidemiological study. Scan $J$ Gastroenterol 1995; 30: 752-57.

5. Fendrick AM, Chernew ME. Hirth RA. Alternative managementsstrategies for patients with suspected peptic ulcer disease. Ann Intern Med 1995; 123:260-68.

6. Hopkins RJ, Girardi LS, Turney EA. Relationship between Helicobacter pylori eradication and reduced duodenal and gastric ulcer recurrence: a review. Gastroenterology 1996; 110: 1244-52.

7. Pilotto A, Franceschi M Valerio G, et al. Helicobacter pylori infection in eldery subjects. Age Ageing 1999:29:103-09. 